apparently healed. Hearing was, for practical purposes, perfect. He had lost all pain and tenderness about the mastoid. Remarls by Mr. SHEILD. - The combination of a large aural polypus and "head symptoms" in a case of longstanding otorrhoca is often difficult to manage. In a large number of such cases there is retention of pus and caseating products of inflammation within the mastoid cells. A soft granulation polypus in a child will often disappear on opening the mastoid and washing out the cavities contained in it. In cases like the present there is always considerable danger of thrombosis of the lateral sinus, and this in itself is a sufficient argument for opening the mastoid. Should there be definite evidence of thrombosis in the deep jugular vein, the question of ligature of that vessel below the thrombosed parts must arise. These cases are desperate, and if general pyæmic infection has already occurred the chance of recovery is small. It would seem generally better to remove the polypus at the same time as the cells are opened. Thus a free exit is afforded through the auditory canal for pus and the irrigated fluid. Should there be definite evidence of necrosis of the mastoid, and the dead bone be deep and fixed, a per. manent opening had better be established behind the ear by the introduction of a small lead or silver tube, for in such cases pus will certainly re-collect. Every effort must be made to destroy the remnants of the polypus, and to obtain the healing of a perforated membrane. If this be accomplished the results may be surprisingly good, as in the present instance, and bearing may for practical purposes be completely restored. In most cases, however, such will not be the case, the ear remaining permanently damaged, and needing long-continued care and attention. Much has been written lately about the operation of opening the mastoid, and the position of the lateral sinus. The divergence of opinion regarding the latter important anatomical consideration leads to the practical conclusion that, as one can never be certain of the exact depth and situation of the sinus in any given case, the utmost caution should always be employed. By the method of removing layer after layer of bone with a mallet and gouge, and carefully inspecting each portion of exposed bone before going deeper, the lateral sinus can hardly be wounded, and the actual entrance into the cells can safely be made with a blunt-pointed director. The free hæmorrhage observed in these cases appears at first sight alarming. It is due to excessive engorgement of the vessels of the inflamed bone, and soon ceases on the application of hot water with the syringe. Doubtless the local depletion is most beneficial, and goes far to aid the striking relief of pain and discomfort which the operation usually effects.

\section{HULL ROYAL INFIRMARY.}

PAPILLOMA OF BLADDER; SUPRA-PUEIC CYSTOTOMY; RECOVERY.

(Under the care of Mr. Craven.)

THE following account of the removal of a tumour from the bladder after cystotomy and of its microscopical characters is of interest and importance. From the duration of symptoms, the small size attained by the growth, and its intimate structure, there is reason for giving a favourable prognosis as to non-recurrence of the disease. We hope that the ultimate result will be given on some future occasion, for growths somewhat resembling this in site and characters have recurred and proved fatal, the recurrences having been of sarcomatous nature. The suprapubic method of operating for the removal of these growths is the one which is found to give the most general satisfaction. For the notes of this case we are indebted to $\mathrm{Mr}$ Frank Savery, house surgeon.

G. W-, a labourer aged sixty-four, was admitted as an in-patient under the care of Dr. Daly on May 15th, 1890. He had enjoyed good health until 1886, and had never had any illness of importance previously to that date. In September, 1886, he was seized quite suddenly with pain in the right lumbar region, and this was followed in a few days by the presence in the urine of some material like "coffee," and then by bright blood. Since that time the urine has never been free from blood. The blood, however, varied considerably in amount; it was generally uniformly mixed with the urine, but sometimes in clots; the clots were not worm-shaped, and the passage of clots was not preceded by pain in the lumbar region. Exercise increasea tbe amount. There was no pain before, during, or after micturition, and no difliculty in micturition except when. clots were present. The freruency of nicturition was not increased; the anount of urine was not increasing. He lost a little weight--about 1 st. in three years. There was. no family history of cancer or new growth.

On admission the patient was a well-nourished man, but very anxemic. He complained of pain in the right lumbar region. The urine was acid, sp. gr. 1020 ; it con. tained albumen and a considerable quantity of blood, the colour being bright red; the albumen was not in excess of the amount of blood; under the microscope blood-corpuscles were seen, but no casts. The urine was more darkly coloured with blood towards the end than at the commencement of micturition. Temperature normal; pulse weak; other organs appeared to be quite healthy. The bladder was sounded, but no stone felt. The prostate was not enlarged. There was no pain or swelling in the renal regions. Per. chloride of iron was given, but the urine remained the same; then hamamelis, with a similar result. Dr. Daly and Mr. Craven considered the blood came from the bladder, probably from a new growth, and, as the patient was evidently going down-hill, advised an exploratory operation. On June 10th, at 9.30 A .I. Mr. Craven operated, being assisted by Mr. Evans. The bladder was washed out, and then moderately distended with boracic lotion. The rectal bag was passed, and filled with eight ounces of water. An incision was then made in the middle line of the abdomen, four inches long, the lower end being at the symphysis. The bladder was opened and explored with the finger, when a small grow th was found on the left side of the fundus near. the opening of the left ureter, and possibly surrounding it. It consisted of several nodules, and had a broad base of attachment. It was removed by means of scissors. There was very little hremorrhage. A few stitches were put in the upper part of the wound, and a drainage-tube, with a long tube attached to it, in the lower, passing into the bladder.

June 12th. - The patient has an attack of bronchitis, and is very bad; he wanders a good deal. All the urine comes. by the wound, and contains blood.

20th.-Urine alkaline (sp. gr. 1020); contains a trace of albumen, but no blood; there is a deposit of pus. $\mathrm{He}$ passes some urine by the penis. The bronchitis is much better, but he is still delirious at night. Ordered boracic acid internally.

26th.-Urine acid, contains a little blood; pus much less. From this time the urine remained free from blood. He. passed more and more urine by the penis, and on July 20 th the wound in the abdomen was quite healed. He slowly recovered, and was discharged on Aug. 8th to a Convalescent Home at Ilkley.

On microscopic examination the tumour had the appearance of a simple adenoma, there being a considerable fibrous stroma, with tubes cut across, lined by a single layer of epithelium.

\section{BEDFORD GENERAL INFIRMARY.}

A CASE OF STRANGULATED HERNIA COMPLICATED WITH PAROTITIS ; RECOVERY:

(Under the care of Mr. KINSEY.)

THrs case is an example of inflammation of the parotid glands following an operation in which not only was the peritoneum implicated, but the testis manipulated to an unusual extent. We published a paper by Mr. Stephen Paget, '" giving the result of his investigations as to parotitis supervening on surgical operations. Of the 101 cases which he had collected, 10 followed injuries or diseases of the urinary tract; 18 injuries or diseases of the alimentary canal ; 23 injuries or inflammation of the abdominal wall, peritoneum, or pelvic cellular tissue ; and 50 injuries, diseases, or operations on the generative organs, 27 of these being operations on the ovary. The chart bears out his observation-that, as a, rule, the parotitis is not accompanied by very severe fever, rigors are rare, and the inflammation does not progress to abscess, unless it forms part of a pycemic infection. For the report of the case we are indebted to Mr. R. H. Elliot, house surgeon.

$G \mathrm{D}$-, aged eighteen, was admitted to the Bedford Infirmary on August 29 th, 1890 , suffering from strangulated 
right inguinal hernia. The hernia, which is then said to having been dressed already on the night of Aug. 30th and have been strangulated, was reduced with difficulty five days on Sept. 1st. On the eighth day acute left parotitis began, ago. The rupture again became irreducible at 8 A.M. of the right side becane affected on the ninth day, the temAug. 29th. His medical man gave opium (?) in the form of perature reaching $102 \cdot 4^{\prime 2}$ on the eleventh day and $103^{\circ}$ a pill, and made two attempts at reduction. The patient on the twelfth day, after which it gradually fell, to reach arrived here at twelve midnight. He had had nausea and normal on the fifteenth day. On the thirteenth day absolute constipation all day, and abdominal pain during the xight side of the face was more swollen, and the the afternoon. There was a strangulated inguinal hernia eyelids on that side were swollen. The left side was also as large as a hen's egg, lying in the scrotum on the right swollen. On the fifteenth day the pain was much less, side, hehind the testicle. Constitutional signs well marked. and the swelling on the left side had almost disappeared, Mr. Kinsey came at 2.15 A.M., and under chloroform cut but was still present on both sides on the nineteenth

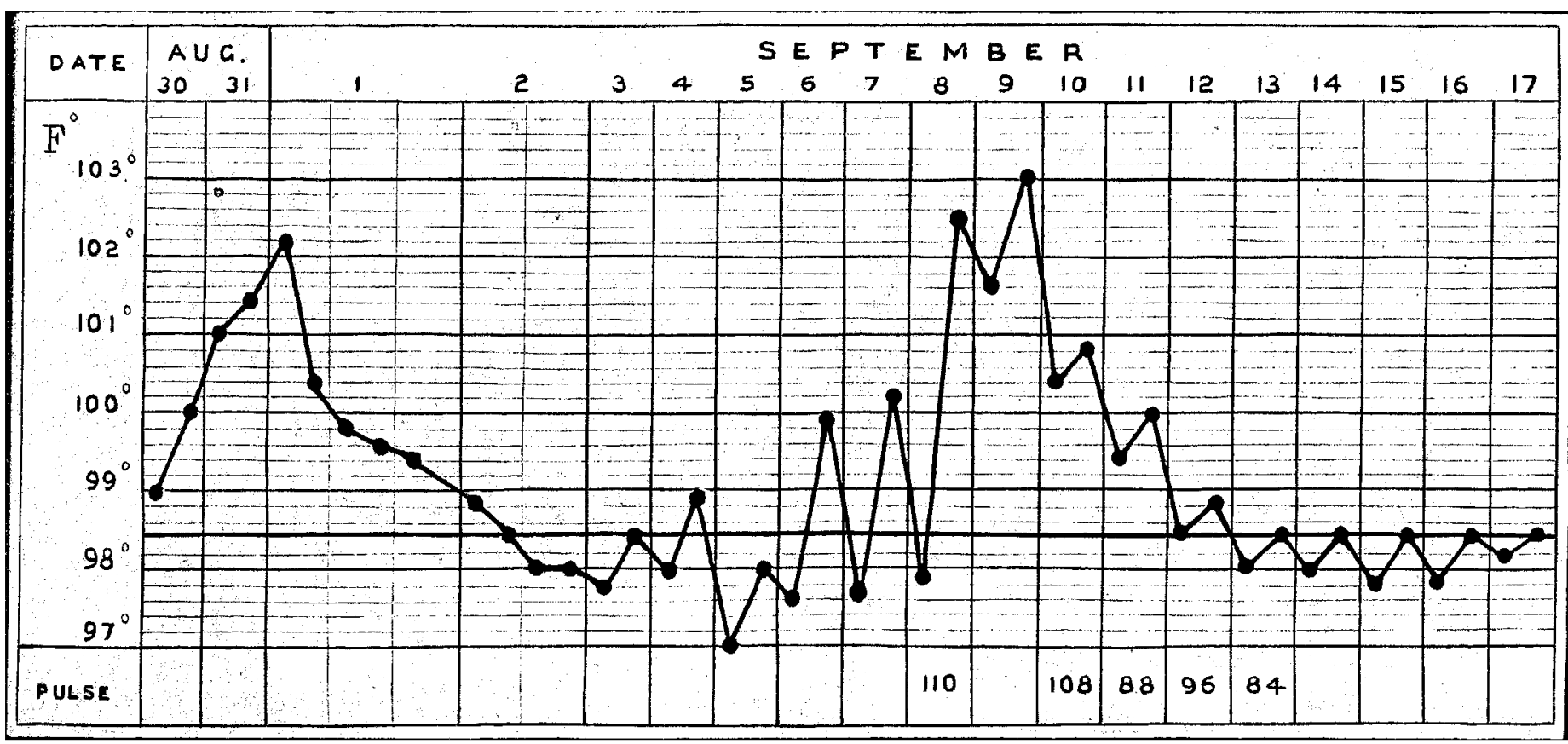

down on the hernia. It was exclusively enterocele, and the strangulating band was long and tight, lying in the neighbourhood of the internal ring. The gut was red and polished. It was returned, and the sac was dissected off from the surrounding parts with considerable difficulty, the dissec tion taking a long time. The temperature rose steadily till it reached $102^{\circ}$ on Aug. 30th, at 10 .. M, from which time it fell and remained normal till Sept. 7th, on which day the bowels acted twice after an enema. The patient gave no real cause for anxiety beyond his temperature, but the wound suppurated freely. It was dressed twice daily after Sept. 4th, day, after which it gradually subsided. There was no albuminuria.

Remarlis by Mr. Elliot.-This case, I think, raises an interesting point of connexion between the testicle and parotid. The position of the testicle, the tediousness of the operation, the suppuration which unfortunately ensued, would probably indicate considerable interference with the cord ; and, on the other hand, the patient could in the fortnight or so preceding admission think of no source from which he could have contracted the mumps, nor did he communicate that disease to his fellow patients, nurses, or others.

\section{Mttrdiat Soctectics.}

\section{PATHOLOGICAL SOCIETY OF LONDON.}

Carcinoma following Lupus of Face-Morphology, Cultivation, and Toxic Products of the Tubercle BacillusTubercular Guinea-pigs under Koch's Treatment.

Av ordinary meeting of this Society was held on Feb. 3rd, the President, Dr. W. H. Dickinson, in the chair.

Mr. E. Solly showed, for Mr. James Berry, a case of Carcinoma following Lupus of Face. The patient was a male aged forty-five, who had been cured of lupus of the face when aged fifteen, and the present cancerous growth commenced only two months ago.

Professor Crookshank made a communication on the Morphology, Cultivation, and Toxic Products of the Tubercle Bacillus (an abstract will be found in another column). This was followed by a demonstration of microscopical preparations and cultivations. The preparations were shown under thirty.four microscopes (including twenty-three one-twelfth objectives). The preparations included tubercle bacilli in sputum and pus from different cases in man; in pure cultivations in glycerine agar-agar, glycerine broth, broth withont glycerine, and glycerine milk; in cream from a tubercular cow; in the liver of fowls, lung of guinea-fowl, lung of ostrich, lung of rabbit, in bovine tubercular mammitis, in miliary tuberculosis in a calf inoculated with human tubercular sputum, in lung of horse, in spleen of tubercular guinea-pig, and several preparations illustrating tubercle bacilli and "phagocytes"-from frogs inoculated with pure cultivations of the bacillus. There were also preparations shown of the crystals obtained from the extract of cultures and of tubercular tissue. More than sixty cultivations of the tubercle bacillus were shown; on glycerine agar-agar of different generations, inoculated April 17th, June 28th, July 22nd, Sept. 15th, Oct. 15th, and Dec. 8th, 1890, and Jan. 14th, 1891; in glycerine broth, broth without glycerine, broth withont glycerine with egg albumin, and glycerine milk, inoculated Nov. 11th, 1890, Nov. 13th, and Nov. 28th, and filtrates of these cultures prepared Dec. 5th and 9th. The chemical tests applied to the glycerine culture filtrate and to Koch's own liquid were also shown.

Drs. Arrahnim and Crooksirank made a joint communication on the efl'sct of Koch's Treatment on Tubercular Guinea-pigs with liquid received by Dr. Abraham direct from Berlin. Two tubercular guinea-pigs had received six injections since Jan. 7 th in centigramme doses. Similar doses were at the same time administered to a healthy guinea pig. As further to control observations, two guineapigs in the same stage of tuberculosis were carefully examined both macroscopically and microscopically after death as a guide to the condition of the internal organs in the cases selected for treatment. The lymphatic glands were caseons, and contained bacilli. There were deposits in the spleen, which also contained bacilli. The cases selected for treatment were therefore undoubtedly tubercular, withont the disease being, in the opinion of the authors, too far advanced. There were well-marked chains of tubercular glands, but the animals were nevertheless active and well nourished. The first injection caused considerable constitutional disturbance, rise of temperature, 